\title{
Evaluation of the outcome of different techniques in management of perioral carcinoma
}

\author{
Sameh M El-Taher, ${ }^{a}$ MD; Hassan H El-Ghandour, $b$ MD
}

a) Department of Plastic \& Reconstructive Surgery, Ain Shams University, Cairo, Egypt.

b) Department of Ear, Nose and Throat (Phoniatrics), Ain Shams University, Cairo, Egypt.

\begin{abstract}
Background: Squamous cell carcinoma (SCC) of the oral cavity is a significant public health issue, which uses a wide range of resources. The anatomic and functional complexity of the oral cavity makes the diagnosis and management of this disease entity extremely challenging. Therapeutic strategies are currently based on a combined surgical and radiological approach. Over the past years plastic surgeons tried to improve the quality of life in patients with oral and perioral cancer, offering new reconstructive options more suitable to achieve better functional and aesthetic results.

Patients and methods: In this study 28 patients with squamous cell carcinoma (SCC) in the oral and perioral region; (they were classified into 3 groups according to the size of tumor excision) were involved. Different reconstructive procedures were applied according to the condition. Post operatively they were evaluated for the functional parameters (speech, chewing, swallowing, sphincter and expression), and aesthetic parameters (assessment of tissue excised, patient and doctor satisfaction).

Results: The statistical analysis demonstrates a significant correlation between the size of resection and the functional outcome in all the investigated parameters $(p=0.05)$, meaning improved functional outcome with the minimal reconstructive procedures in all the main anatomical functional areas.

Conclusion: A great effort was made to create a scoring system for both functional and aesthetic outcomes which is very helpful for preoperative planning, excision and reconstruction of the resulting defect. It is considered as a good indicator for improved rehabilitation efficiency through follow up period.
\end{abstract}

\section{Introduction:}

The lips play an important role in emotional and verbal communication. It also esthetically serves as a focal point that defines an individual's unique facial identity. Thus, lip loss or major deformity produces a devastating alteration of normal life. ${ }^{1}$ Oral squamous cell carcinoma (SCC) is the sixth most common malignancy and is a major cause of cancer morbidity and mortality worldwide. Globally, about 500.000 new oral and pharyngeal cancers are diagnosed annually, and three quarters of these are from the developing world. ${ }^{2}$ In Egypt, head and neck cancers represent $3.3 \%$ of all cancers. ${ }^{3}$ Squamous cell carcinoma of the orofacial region is a significant problem in our patient population due to occupational and socioeconomic factors. The disease usually presents in a relatively advanced stage. 4,5

Therapeutic strategies are currently based on a combined surgical and radiologic approach; recent progress in reconstructive surgery has largely improved the quality of life in patients with perioral cancer, offering new reconstructive options more suitable to the complex anatomy and function of the perioral and oral cavity. Thus surgery could be curative as the biological behavior of these cancers is usually locoregionally invasive. ${ }^{6}$ The reconstruction of perioral defects following ablation of cancer has been a challenge for oral and maxillofacial surgeons. The goals of 
perioral reconstruction are esthetic and functional, with oral competence and good lip control. ${ }^{7}$ The purpose of this study was to correlate the outcome (functional and esthetic sequelae in patients treated surgically with or without radiotherapy for perioral tumors.

\section{Patients and methods:}

Between June 2005 and June 2008, in the Plastic and Reconstructive Surgery Department, Ain Shams University Hospitals, 28 patients underwent squamous cell carcinoma (SCC) excision and reconstruction using different types of flaps. The mean age of patients was 33.5 years (range $10-76$ years). Twelve patients $(42.8 \%)$ were females and sixteen patients (57.2\%) were males. Eighteen patients (64.4\%) were smokers. Eight patients $(28.5 \%)$ gave history of precancerous conditions (e.g.leukoplakia of lower lip mucosa, ulcer...etc). Examination of the lymph node affection (using sentinel lymph node biopsy or radio labeling it) revealed eight patients $(28.5 \%)$ to have positive sentinel lymph node. 8,9

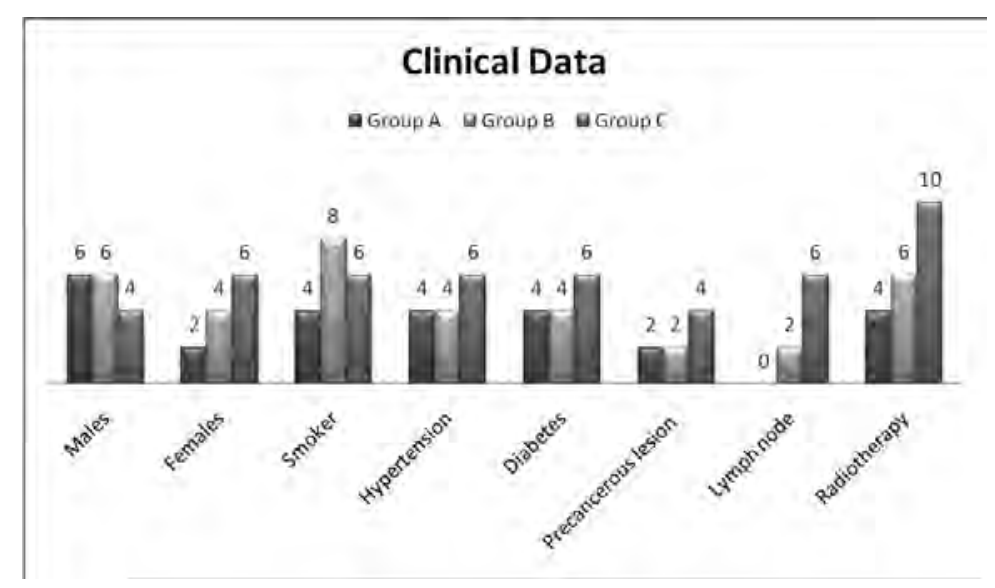

Chart (1): Data collection for patients in the study.

Preoperative evaluation of the patients was performed in all cases where all the tumors were documented to be squamous cell carcinoma using incisional biopsy. Photographing and consent were taken. Patients with medical problems e.g. hypertensive, diabetic, hypercholesterolemia were not excluded from this study.

Frozen section was done for all cases preoperatively then results were later on confirmed by paraffin section examination. The patients were divided into three groups: Group A; included eight patients (28.6\%) with lip defect not exceeding 1/3 of the lip, Group $\mathrm{B}$; included ten patients (35.7\%) with lip defect greater than $1 / 3$ of the lip but less than two thirds. Group C; included ten patients (35.7 $\%$ ) with whole lip defect together with excision of adjacent structures e g. mandible, floor of the mouth and tongue.

\section{Reconstructive procedures:}

Many techniques have been used for reconstruction of the defects according to the size and site of the defect. Generally, defects involving less than a third of the lower lip can be closed primarily (Group A). For larger defects, between one third and two thirds of the lip, Estlander, Burnard burrow, Abo Elfilat and Karapandzic flaps ${ }^{10,11}$ have been used (Group B). However, free tissue transfer has been used for complex total lip reconstruction or when the tumors extended to the oral cavity or the mandible. The most popular distant flap used is the radial forearm flap,, 12 and for cases with combined defect in the mandible free vascularized fibula flap is used (Group C). ${ }^{13}$ 

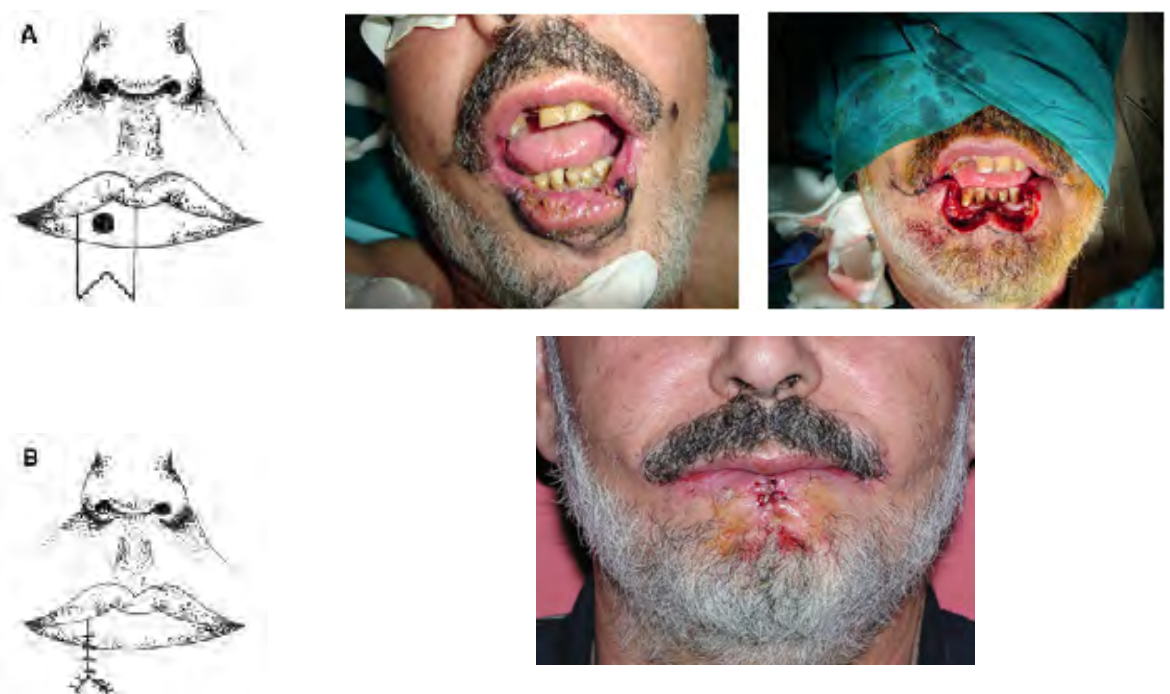

Figure (1): A) Wedge resection with a W excision. B) Closure of W excision.
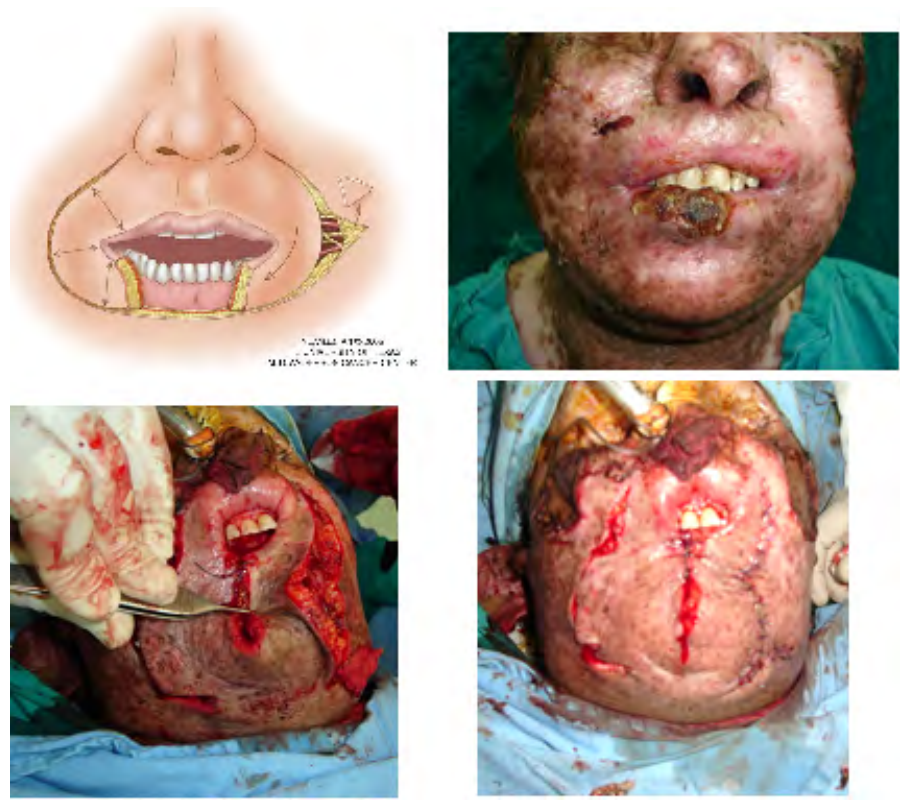

Figure (2): Schematic of Karapandzic flaps.
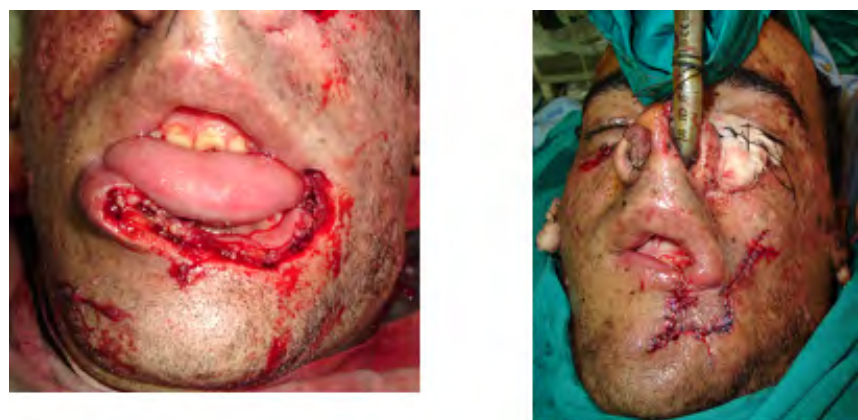

Figure (3): Estlander's lateral flap from the upper lip rotated around the commisure. 


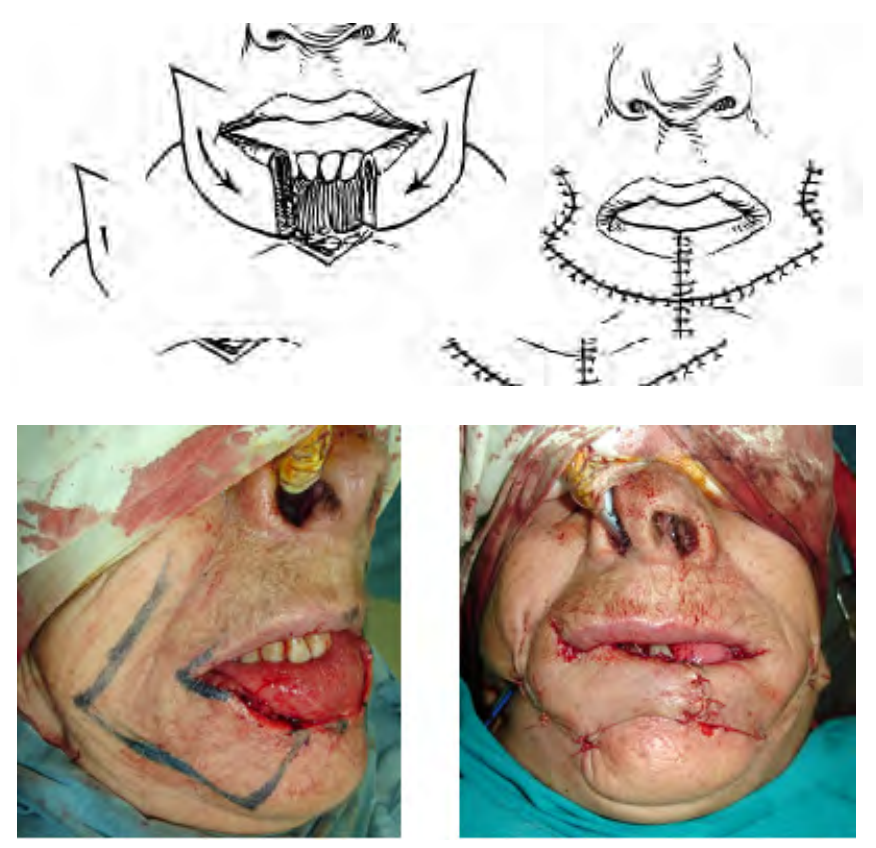

Figure (4): The Gillies fan flap technique.
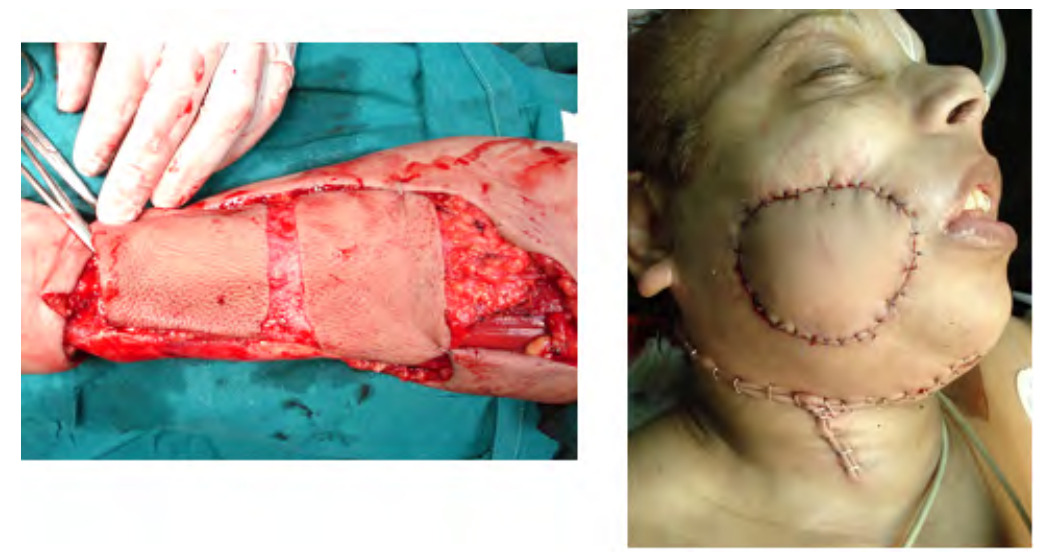

Figure (5): Markings for radial forearm fasciocutaneous free flap with reconstruction of cheek defect after fulthickness tumor excision.

\section{Scoring system:}

To conduct our study we developed a scoring system, which assessed the extent of excision and effect of different reconstructive modalities on restoration of normal action of the perioral region. It was divided into functional and aesthetic parameters each was given a grade from 1-5 according to the effect seen. These parameters were either functional, which included assessment of speech, chewing, swallowing, sphincter and expression; or aesthetic, which included assessment of tissue excised, patient and doctor satisfaction Table(1).

Speech, chewing and swallowing were the functional parameters investigated for a period of two years where patients were scored monthly and the results were checked clinically with each follow-up visit. Assessment of speech quality was done by 'Functional Intraoral Glasgow Scale' self-questionnaire which is an ordinal five-grade scale questionnaire, with high values indicating good speech and 'Conversational Understandability Test' where voiceless fricative segments, $|\mathrm{s}|,|\mathrm{sh}|,|\mathrm{f}|$, and |th|, were used for analysis.

Assessment of chewing and swallowing was done by a five-point ordinal scale selfquestionnaire developed by the Plastic and Reconstructive Surgery Department at Cannies Burn Hospital to allow patients to self-assess chewing and swallowing. Patients scored themselves monthly, and the results were checked at the time of discharge from the individual postoperative rehabilitation program.

Sphincter function was evaluated as regard the air, water and solid whether it is competent or not. Facial expression was assessed by the 
ability to whistle or smile properly. As for the aesthetic parameters; the amount and extent of excision was evaluated according to the thickness of the tissue removed. Finally the patient and doctor satisfaction for the final outcome were also measured.

Table (1): Scoring system sheet.

\begin{tabular}{|c|c|c|c|}
\hline & & Condition & Score \\
\hline \multirow{23}{*}{$\begin{array}{c}\text { Functional } \\
\text { Intra- oral } \\
\text { scale }\end{array}$} & \multirow{5}{*}{ Speech } & Always understandable & 5 \\
\hline & & Needing sometimes repetitions & 4 \\
\hline & & Needing many times repetitions & 3 \\
\hline & & Understandable only by relatives & 2 \\
\hline & & Incomprehensible & 1 \\
\hline & \multirow{5}{*}{ Chewing } & Always understandable & 5 \\
\hline & & Needing sometimes repetitions & 4 \\
\hline & & Needing many times repetitions & 3 \\
\hline & & Understandable only by relatives & 2 \\
\hline & & Incomprehensible & 1 \\
\hline & \multirow{5}{*}{ Swallowing } & Any food without difficulty & 5 \\
\hline & & Solid food with difficulty & 4 \\
\hline & & Semisolid food only & 3 \\
\hline & & Liquid only & 2 \\
\hline & & Swallowing impossible & 1 \\
\hline & \multirow{4}{*}{ Sphincter } & Air Tight & 4 \\
\hline & & Water Tight (Drooling of saliva) & 3 \\
\hline & & Solid Tight & 2 \\
\hline & & Incompetent & 1 \\
\hline & \multirow{4}{*}{ Expression } & Whistling & 4 \\
\hline & & Smile Properly & 3 \\
\hline & & Smile Improperly & 2 \\
\hline & & In able to Smile & 1 \\
\hline \multirow{12}{*}{$\begin{array}{l}\text { Aesthetic } \\
\text { Intra- oral } \\
\text { scale }\end{array}$} & \multirow{4}{*}{$\begin{array}{c}\text { Patient } \\
\text { Satisfaction }\end{array}$} & Excellent & 4 \\
\hline & & Good & 3 \\
\hline & & Fair & 2 \\
\hline & & Poor & 1 \\
\hline & \multirow{4}{*}{$\begin{array}{c}\text { Doctor } \\
\text { Satisfaction }\end{array}$} & Excellent & 4 \\
\hline & & Good & 3 \\
\hline & & Fair & 2 \\
\hline & & Poor & 1 \\
\hline & \multirow{4}{*}{ Excision } & Skin only & 4 \\
\hline & & Full thickness lip & 3 \\
\hline & & Muscle & 2 \\
\hline & & Bone and oral cavity & 1 \\
\hline
\end{tabular}




\section{Results:}

The time interval between surgical treatment and the end of follow up ranged from 2 to 12 months (mean 7 months). The scoring system and analysis of results are summarized Table(2). The total score was then compared to a reference range as follows:

- 31-35----> Excellent, it was found in 6 patients.

- 25 - 30 ---> good, it was found in 10patients.

- 17 - 24 ---> Fair, it was found in 10 patients.

- 8 - 16 ---> Poor; it was found in 2 patients.

By comparing chewing and swallowing with speech, we observed a substantially different trend, meaning a better postoperative recovery of speech in (Group A and B). While in Group $C$ there were significant differences as demonstrated by the different reconstructive modalities. A statistically significant difference was demonstrated for chewing and swallowing $(p=0.05)$ between the patients treated and those not treated with radiotherapy, meaning a worse functional outcome in those patients treated with radiotherapy.

The chi-square distribution demonstrated a statistically significant correlation between the size of resection and the functional outcome with all the assessment methods (Functional Intraoral Glasgow Scale ( $p=0.05$ ); Aesthetic
Intraoral Glasgow Scale, $(\mathrm{p}=0.05)$ meaning for example better speech quality with smaller excisions. A similar statistically significant correlation was also demonstrated between the reconstruction modalities and the functional results by all the tests (Functional Glasgow Intraoral Scale), generally meaning that the less demanding reconstructive procedures had the better functional outcome.

Some statistically significant differences among the reconstruction modalities were demonstrated $(\mathrm{p}=0.05)$ in the large resections involving the floor of the mouth. The simple direct suture gave a better functional outcome than flap reconstruction.

Within all flaps, those that provided bone reconstruction gave better speech quality. In the bilateral small resections of the floor of the mouth, the Functional Intraoral Glasgow Scale $(p=0.05)$ demonstrated statistically significant differences within the reconstruction modalities: the best results were observed in patients who underwent reconstruction using a local flap. The Conversational Understandability Test also showed some differences, but with low statistical significance. The trend was for the less demanding procedure to yield the better functional outcome.

Table (2): Assessment of the different functional and aesthetic results in every patient.

\begin{tabular}{|c|c|c|c|c|c|c|c|c|c|c|c|c|c|c|c|c|c|c|c|c|c|c|c|c|c|c|c|c|c|}
\hline & & $\overline{1}$ & 2 & $\overline{3}$ & 4 & 5 & $\overline{76}$ & 7 & $\overline{8}$ & $\overline{9}$ & $\overline{10}$ & 11 & 12 & 13 & 14 & $\overline{15}$ & $\overline{16}$ & $\overline{17}$ & $\overline{18}$ & 19 & 20 & 21 & 22 & 23 & 24 & $\overline{25}$ & $\overline{26}$ & 27 & 28 \\
\hline \multirow{5}{*}{$\begin{array}{l}\text { Eunctional } \\
\text { Inrat onal } \\
\text { scale }\end{array}$} & Speech & 5 & 5 & 2 & 5 & 3 & 3 & 3 & $\overline{5}$ & 4 & 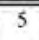 & 3 & 4 & 5 & 4 & 5 & $\overline{c 5}$ & 2 & 5 & 3 & 3 & 3 & $\overline{5}$ & 4 & 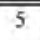 & 3 & 4 & 5 & 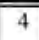 \\
\hline & $\begin{array}{l}\text { Chewing } \\
\end{array}$ & 5 & 5 & 3 & 5 & 2 & 3 & 2 & 5 & 3 & 5 & 3 & 4 & 4 & 3 & 5 & 5 & 3 & 5 & 2 & 3 & 2 & 5 & 3 & 5 & 3 & 4 & 4 & 3 \\
\hline & Swallowing & 4 & 5 & 3 & 5 & 2 & 3 & 2 & 5 & 4 & 5 & 2 & 4 & 4 & 4 & 4 & 5 & 3 & 5 & 2 & 3 & 2 & 5 & 4 & 5 & 2 & 4 & 4 & 4 \\
\hline & Sphincter & 3 & 4 & 2 & 3 & 2 & 2 & 2 & 4 & 3 & 3 & 2 & 3 & 4 & 4 & 3 & 4 & 2 & 3 & 2 & 2 & 2 & 4 & 3 & 3 & 2 & 3 & 4 & 4 \\
\hline & Expression & 4 & 4 & 2 & 4 & 2 & 2 & 2 & 4 & 3 & 4 & 2 & 3 & 3 & 3 & 4 & 4 & 2 & 4 & 2 & 2 & 2 & 4 & 3 & 4 & 2 & 3 & 3 & 3 \\
\hline \multirow{3}{*}{$\begin{array}{l}\text { Aesthetic } \\
\text { Intra-oral } \\
\text { scale }\end{array}$} & $\begin{array}{c}\text { Patient } \\
\text { Satisfaction }\end{array}$ & 3 & 3 & 2 & 4 & 2 & 3 & 2 & 3 & 3 & 3 & 3 & 3 & 3 & 3 & 3 & 3 & 2 & 4 & 2 & 3 & 2 & 3 & 3 & 3 & 3 & 3 & 3 & 3 \\
\hline & $\begin{array}{c}\text { Doctor } \\
\text { Satisfaction }\end{array}$ & 3 & 4 & 3 & 4 & 2 & 2 & 2 & 4 & 3 & 3 & 2 & 4 & 3 & 3 & 3 & 4 & 3 & 4 & 2 & 2 & 2 & 4 & 3 & 3 & 2 & 4 & 3 & 3 \\
\hline & Excision & 3 & 3 & 1 & 4 & 2 & 1 & 1 & 4 & 2 & 2 & 1 & 2 & 3 & 3 & 3 & 3 & 1 & 4 & 2 & 1 & 1 & 4 & 2 & 2 & 1 & 2 & 3 & 3 \\
\hline \multicolumn{2}{|c|}{ Total score } & 30 & 33 & 18 & 35 & 17 & 18 & 16 & 34 & 25 & 30 & 18 & 27 & 29 & 27 & 30 & 33 & 18 & 35 & 17 & 18 & 16 & 34 & 25 & 30 & 18 & 27 & 29 & 27 \\
\hline
\end{tabular}




\section{Discussion:}

Several studies aiming to evaluate functional outcome after perioral cancer surgery were carried out in the past, and in most cases, only a correlation to broad factors could be statistically demonstrated. In all anatomical areas of the perioral and oral cavity, the general pattern was for consistently better speech quality and good functional outcome with smaller excisions. Unilateral resections gave a better functional outcome than those corresponding in size and involving the anterior midline in the anterior floor of the mouth. ${ }^{14}$, 15,16

Different modalities in management of oral and perioral carcinomas play an important role in the functional \& aesthetic outcome; 17,18

- Any disruption in the muscle of the lip or any affection of the sphincteric action leads to disturbance in the speech articulation.

- The integrity of the mandibular insertions of the genioglossus and geniohyoid muscles is likely to be crucial in providing adequate tongue dynamic stability and active motion, as loss of integrity severely impairs speech articulation. $^{7}$

- The tongue as it is the primary active articulator producing the voiceless fricative segments $|\mathrm{s}|,|\mathrm{sh}|,|\mathrm{f}|$, and $|\mathrm{th}|$.

- The soft palate in the posterior oral cavity: all resections involving the soft palate gave a remarkable deterioration in speech that was always attributable. ${ }^{16}$

In this study, the main goal was to produce simultaneously good functional and aesthetic outcome, we consider excision when affecting the functional unit or the sphincter action, so we tried to limit its size as much as we can by proper intra-operative frozen section technique. Then improving the shape of the resulting scar, by hiding them in the edges of the facial aesthetic units, proper correction of muscle after excision to give a proper tone and correction of the sphincter by sphincterplasty to correct microstomia.

Also post operative rehabilitation by speech therapy, must be considered. Then a subjective assessment was done by the patients. Most of them (Group A and B) had good satisfactory results due to the small sized tumor which could be handled easily due to the wide variety of options we had. On the contrary, patients in
(Group C) showed fair satisfactory results as they had major reconstructive operation which obviously couldn't be much improved (Safety better than cosmoses).

We investigated speech by the original two subjective assessment methods (Conversational Understandability Test and Functional Intraoral Glasgow Scale), 18 to accurately measure its actual dispersion after perioral cancer surgery. A statistically significant association of all voice quality measures stronger between the two tests proving this association to be the most suitable investigation method for speech.

In our experience, the reconstruction modalities do not seem to influence the overall speech quality, as overall speech quality is related mainly to the extent of surgical excision. Nevertheless, the role of bone reconstruction of the mandible after full thickness resection is relevant in the ability to pronounce voiceless fricative sound. Patients treated with radiotherapy had worse functional outcomes. This was generally because of the larger extent of the surgical excision and radiotherapy itself was found to play a more individual and specific role in decreasing speech quality.

Chewing and swallowing are life-supporting functions of the oral cavity that are closely related to nutrition. The complete loss of swallowing is a certain life-threatening condition, whereas the complete loss of chewing can be somewhat compensated for by appropriate food preconditioning. ${ }^{16,} 19$

Swallowing occurs in three stages. The first is voluntary contraction of the mylohyoid muscles, and the other two are reflexly produced by the base of the tongue, the soft palate, the larynx, the posterior pillars of the fauces, and the pharynx. In our sample, a complete loss of swallowing occurred only in those patients who had large excision of the base of the tongue and of the retromolar trigone, with a massive ablation of those anatomical structures that reflexly produced swallowing. ${ }^{18}$

A slightly lesser degree of severe functional impairment, but never a complete loss of swallowing, occurred in a relevant number of patients who had a large full thickness resection of the anterior floor of the mouth. In these patients, the complete ablation of the anterior floor of the mouth and the anterior tongue 
obviously harmed the active voluntary phase of swallowing. ${ }^{20}$

Mastication is a complex process resulting from fine and coordinated movements of the mandible at the temporomandibular joint carried out by four main muscles and three secondary ones sitting in the floor of the mouth. Therefore, the act of chewing is allowed by the anatomical functional integrity of active structures, such as muscles, and passive structures, such as the mandibular lever, teeth, mucosal lining, and salivary glands. ${ }^{16,} 21$

In our sample, ablative surgery almost didn't include the main muscles of mastication but only involved the secondary ones and passive structures such as mucosa, salivary glands, teeth, and mandibular bone. A complete loss of chewing occurred only in the wide resections of the anterior and lateral floor of the mouth and in the large resections of the retromolar trigone. Nevertheless, a degree of chewing impairment occurred in almost all patients in Group C.

We observed only slight differences in the eventual outcome for both functions between small and medium-sized resections of the floor of the mouth. These data can be explained by the ability of the remaining anatomical active structures to take charge of the excised muscles' function. This concept is confirmed by the remarkable differences in functional efficiency, both for chewing and swallowing.

In the general sample, radiotherapy seems to be a negative prognostic factor for both functional and aesthetic outcome: an explanation for this could be the routine combined use of radiotherapy and surgery in the larger tumor resections. ${ }^{22}$

Speech showed a more complete recovery than swallowing and chewing after surgery for perioraloral cancer. This may be attributed to the higher cortical control of speech, large reflex controlled functions, that allows for more chances of success for the rehabilitation therapy and the tendency for the patients to subjectively overestimate the quality of their speech, whereas chewing and swallowing would be less liable to subjective interpretation. ${ }^{15,16,17}$

\section{Conclusion:}

In our experience we could correlate the functional and aesthetic outcome to different surgical modalities and its affection on the specific anatomical perioral region. A great effort was made to create a scoring system for both functional and aesthetic outcomes which is very helpful for preoperative planning and avoiding, marled tissue excision during surgery and is considered as a good indicator for improved rehabilitation efficiency through follow up period.

\section{References:}

1- Negri E, La Vecchia C, Levi F, Franceschi S, Serra-Majem L, Boyle P: Comparative descriptive epidemiology of oral and oesophageal cancers in Europe. Eur $J$ Cancer Prev 1996; 5: 267-279.

2- Neville BW, Day TA: Oral cancer and precancerous lesions. CA Cancer J Clin 2002; 52(4): 195-215.

3- El-Hattab OH, Nouh MA: Epidemiology of cancer. Cairo Univ Press 1998; 1: 1-6.

4- Orringer JS, Shaw WW, Borud LJ, et al: Total mandibular and lower lip reconstruction with a prefabricated osteocutaneous free flap. Plast Reconstr Surg 1999; 104(3): 793-797.

5- Canto MT, Devesa SS: Oral cavity and pharynx cancer incidence rates in the United States, 1975-1998. Oral Oncol 2002; 38(6): 610-617.

6- Palm CA, Guilane PJ, Gilbert RW: Current treatment options in squamous cell carcinoma of the oral cavity. Surg Oncol Clin N Am 2004; 13(1): 47-70.

7- Yamauchi M, Yotsuyanagi T, Yokoi K, et al: One-stage reconstruction of a large defect of the lower lip and oral commissure. Br J Plast Surg 2005; 58: 614-618.

8- Ionna F, Chiesa F, Longo F, et al: Prognostic value of sentinel node in oral cancer. Tumori 2002; 88(3): 18-19.

9- Ross G, Shoaib T, Soutar DS, et al: The use of sentinel node biopsy to upstage the clinically N0 neck in head and neck cancer. Arch Otolaryngol Head Neck Surg 2002; 128: 1287-1291. 
10-Karapandzic M: Reconstruction of lip defects by local arterial flaps. Br J Plast Surg 1974; 27(1): 93-97.

11-Rashid M, Hanif MS, Illahi I, et al: Reconstruction of lip defects with the Karapandzic technique. J Coll Physicians Surg Pak 2003; 13(4): 219-222.

12-Ozdemir R, Ortak T, Kocer U, et al: Total lower lip reconstruction using sensate composite radial forearm flap. $J$ Craniofacial Surg 2003; 14(3): 393-405.

13-Wechselberger G, Gurunluoglu R, Bauer T, Piza- Katzer H, Schoeller T: Functional lower lip reconstruction with bilateral cheek advancement flaps: Revisitation of Webster method with a minor modification in the technique. Aesthetic Plast Surg 2002; 26(6): 423-428.

14-Harrison LB, Sessions RB, Hong WK: Head and Neck Cancer: A Multidisciplinary Approach. Philadelphia, Pa: Lippincott (Publisher); 1999.

15-Schliephake H, Jamil MU: Impact of intraoral soft-tissue reconstruction on the development of quality of life after ablative surgery in patients with oral cancer. Plast Reconstr Surg 2002; 109: 421.

16-Nicoletti G, Soutar DS, Jackson M, Wrench A, Robertson G, Robertson C: Chewing and swallowing after surgical treatment for oral cancer: Functional evaluation in 196 selected cases. Plast Reconstr Surg 2004; 114(2): 329-338.

17-Knezevic P, Uglesic V, Virag M, Varga S: Comparison of three methods of oral reconstruction for quality of life assessment. Ann Plast Surg 2002; 48: 334.

18-Nicoletti G, Soutar DS, Jackson M, Wrench A, Robertson G, Robertson C: Objective assessment of speech after surgical treatment for oral cancer: Experience from 196 selected cases. Plast Reconstr Surg 2004; 113: 114.

19-Verschuur HP, Irish JC, O'Sullivan B, Goh C, Gullane PJ: A matched control study of treatment outcome in young patients with squamous cell carcinoma of the head and neck. Laryngoscope 1999; 109: 249-258.

20-Gullane PJ: Changing concepts in soft tissue repair of oral and oropharyngeal. Arch Otolaryngol Head Neck Surg 2000; 126: 912-913.

21-Bundgaard T, Tandrup O, Elbrond O: A functional evaluation of patients treated for oral cancer: A prospective study. Int J Oral Maxillofac Surg 1993; 22: 28-34.

22- Langstein HN, Robb GL: Lip and perioral reconstruction. Clin Plastic Surg 2005; 32 : 431-445. 